\title{
The Effect of Copper Ions on Colour in Extracts of Cabernet Sauvignon and Sangiovese Skins Under Wine-like Conditions
}

\author{
L. Rustioni
}

Università degli Studi di Milano, CIRIVE - Centro Interdipartimentale di ricerca per l'innovazione in Viticoltura ed Enologia, via G. Celoria 2, I-20133 Milano, Italy

Submitted for publication: June 2015

Accepted for publication: August 2015

Key words: Grape pigments, coloured complexes, metals, interactions, malvidin

Pigment-metal complexes are generally not considered to play significant roles in grape and wine colour. However, to the best of my knowledge, this hypothesis has never been investigated. In this work, grape skin extracts (cultivars Sangiovese and Cabernet Sauvignon) were allowed to react with copper sulphate aqueous solutions at different concentrations. High, and toxic, copper additions produced significant absorption increases in the green spectral region (corresponding to the typical absorption band of anthocyanins). Further studies will be necessary to characterise the nature of this interaction.

\section{INTRODUCTION}

In grapes and wines, phenolics do not exist in isolation, but are surrounded by other compounds able to interact with them. Tannin interactions have been widely studied, but they are still an interesting research subject (Hanlin et al., 2010; Rustioni et al., 2014a). The impact of anthocyanin interactions on grape and wine colour has been summarised by Boulton (2001) in his review, providing the basis for further studies involving new methods (Di Meo et al., 2012) or for considering the equilibrium between different cofactors (Rustioni et al., 2012).

Flavonoids (such as proanthocyanidins and anthocyanins) bind metals through complexation involving their ortho-diphenol groups (Boulton, 2001; Dixon et al., 2005). The influence of metals in anthocyanin colour has been known a long time. Shibata and Shibata proposed the metal complex theory in 1919, according to which the blue colour of some flowers is produced by a complex of anthocyanins and metal ions, such as magnesium and calcium (Kondo et al., 1992; Takeda, 2006). In 1931, Robinson and Robinson suggested the need to "test the response to the ferric reaction" in their survey of anthocyanins and, according to Harborne (1958), "the effect of aluminum chloride on the spectra of anthocyanins has been described as distinguishing pigments containing o-dihydroxyl groups". The contribution of magnesium to the formation of commelinin (a blue metal-anthocyanin complex) was demonstrated by Takeda and Hayashi (1977). Takeda (1977) also tested complexes with a number of elements (K, Ca, Sr, Ba, Mn, Co, Ni, Cu, Zn, Cd, Hg, Al, Fe and $\mathrm{Mg}$ ), obtaining different colours in the reaction mixtures (purple, blue and greenish blue). He concluded that Mn, Co, $\mathrm{Ni}, \mathrm{Zn}$ and $\mathrm{Cd}$ were capable of replacing the $\mathrm{Mg}$ contained in the molecule of the authentic commelinin to form crystalline blue complex molecules, which are homologous. Commelinin is readily soluble in water, cannot be dialysed, and the blue colour is stable in concentrated solutions. When diluted, the solution quickly becomes colourless due to decomposition, suggesting that commelinin is an associated supra molecule held together by weak hydrophobic interactions (Kondo et al., 1992). Kondo et al. (1992) described the commelinin structure, demonstrating that, in Commelina communis L., three typical mechanisms for flower colour development and stability co-exist: metal-complexation, self-association and co-pigmentation. The association of flavonoids is due to the hydrophobic interactions of the aromatic nucleus of anthocyanins (malonylawobanin) and flavones (flavocommelin), and possibly also compression due to external hydrogen bonding between the sugars and water to form commelinin, low molecular mass components gather together with high specificity.

The blue colour of blueberries (Vaccinium spp.) is associated with aluminium complexes of otherwise red anthocyanins. However, the ability to form such complexes is related to an ortho-dihydroxyl arrangement on the B ring, so that, while cyanidin, delphinidin and petunidin can form them, those of malvidin, pelargonidin and peonidin cannot. The role of polyphenols has been demonstrated in copper complexation in red wines (Vasconcelos et al., 1999) and, since malvidin-3-O-glucoside is the major anthocyanin in most vinifera grapes, its role in the colour of wines is not

*Corresponding author: laura.rustioni@unimi.it [Tel.: 003902503 16556]

Acknowledgements: I would like to thank Prof. Osvaldo Failla, for the precious support and helpful collaboration in my research activities 
considered significant (Boulton, 2001).

Copper sulphate is widely used in viticulture and oenology. Downy mildew (Plasmopara viticola), one of the most serious diseases of grapevine worldwide, is controlled mainly by sprays with copper compounds (also allowed in organic farming), despite their unfavourable ecotoxicological profile (Dagostin Schärer et al., 2011). Moreover, this salt is traditionally used to remove the "reduction" smell in wine making.

This preliminary experiment aimed to study the effect of copper on the colour of grape anthocyanins in model wine solutions, testing two cultivars with different pigment profiles: one with a high percentage of ortho-dihydroxylated molecules (Sangiovese), and the other with a typical malvidin-rich profile (Cabernet Sauvignon).

\section{MATERIALS AND METHODS}

Cabernet Sauvignon and Sangiovese grapes were collected in the germplasm collection vineyard of the Università degli Studi di Milano (Lombardy region, northern Italy), already described in Rustioni et al. (2013a). Ripe grapes were sampled in 2008 and frozen $\left(-20^{\circ} \mathrm{C}\right)$ until extraction (performed within three months). Three replications of 20 skins for each cultivar were extracted in $50 \mathrm{ml}$ of a model wine solution $(3,5$ $\mathrm{g} / \mathrm{L}$ tartaric acid, $100 \mathrm{mg} / \mathrm{L}$ sodium metabisulphite, $12.5 \%$ ethanol, buffered at $\mathrm{pH} 3.2$ by $\mathrm{NaOH}$ ) overnight. Then, $3 \mathrm{ml}$ of extract was reacted with $1 \mathrm{ml}$ of aqueous solutions of five different cupric sulphate concentrations. For each extract, a blank was obtained by a pure water dilution, maintaining the proportion 3:1 (called copper sulphate concentration 0). To be aware of possible cupric sulphate salt interference in the solution's optical properties, the pure model wine solvent was also diluted by each cupric sulphate concentration solution, keeping the proportion $3: 1$.

Copper sulphate solution was prepared considering the toxic legal limit of copper concentration in wines at $1 \mathrm{mg} / \mathrm{l}$, and serial concentrations (factor of 10). With the object to reproduce the wine stoichiometric equilibrium, the copper sulphate concentration was calculated by considering the copper mass in relation to the molecular weight of the copper sulphate pentahydrate used in this experiment. Because the average weight of 20 whole berries was $30 \mathrm{~g}$, and the relative extracting solvent was $50 \mathrm{ml}$, the copper sulphate pentahydrate concentration was multiplied by $5 / 3$. Finally, considering the dilution caused by the proportion between the skin extracts and the cupric sulphate solution (3:1), I calculated $8.73 \mathrm{mg} / \mathrm{l}$ as the concentration of $\mathrm{CuSO}_{4} \times 5 \mathrm{H}_{2} \mathrm{O}$ necessary to obtain the wine stoichiometric equilibrium of the maximum legal copper concentration (called copper sulphate concentration 1). However, to evaluate the possible complexation effect at higher (and toxic) concentrations, I also prepared solutions of the same salt that were 10 (called copper sulphate concentration 2), 100 (3), 1000 (4) and 10000 (5) times more concentrated.

All reagents and solvents were purchased from SigmaAldrich Co. All the mixtures were reacted for 18 hours.

Spectrophotometric analyses were performed using a JASCO 7800 spectrophotometer (JASCO, Mary's Court, Easton, Maryland). This was set up to record the extract absorption between $420 \mathrm{~nm}$ and $700 \mathrm{~nm}$, in $1 \mathrm{~nm}$ steps. Just before analysis, the necessary dilutions were obtained by using the same model wine solution:water (3:1) solvent, keeping the absorbance maxima lower than 1 . Then each spectrum was multiplied by the relative dilution factor.

All data were statistically analysed using SPSS $®$ statistical software (Version PASW Statistics 22, SPSS Inc, Chicago, Illinois). The spectrum of each group was compared by delineating the confidence interval $(95 \%)$ of each recorded wavelength. The increasing ratio of the absorption intensity was tested statistically by a general linear model. Cultivar means were compared by way of the LSD test $(\mathrm{P}=0.05)$.

\section{RESULTS AND DISCUSSION}

Fig. 1 reports the cupric sulphate absorbance properties at the studied concentrations in the experimental solvent. Low concentrations (1 to 3 ) produced undetectable differences

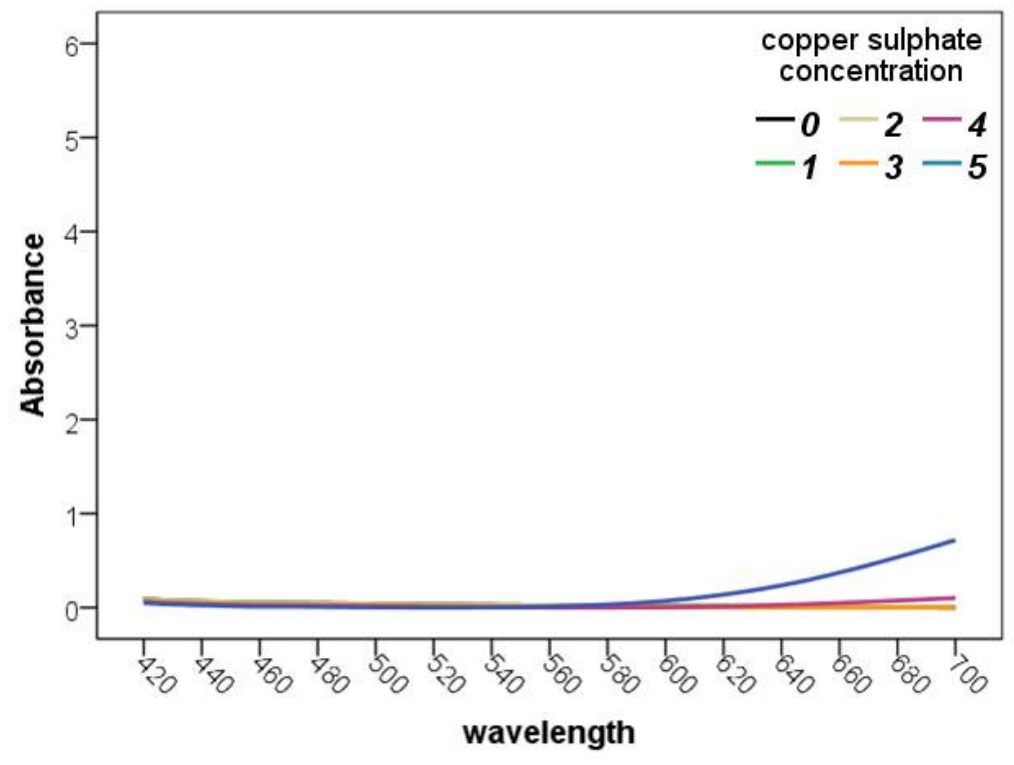

FIGURE 1

Cupric sulphate absorbance properties at the studied concentrations ( 0 to 5$)$ in the experimental solvent. 
when compared to the blank. Considering concentration 5 and, to a lesser extent, 4, it was possible to highlight the copper sulphate absorption tail in the red spectral region. In fact, copper sulphate solution at concentration 5 appeared lightly blue in colour.

Figure 2 reports the copper sulphate effect on the grape extract colour. The absorption band in the green region (maxima around $525 \mathrm{~nm}$ ) represents the typical anthocyanin pigmentation. High copper sulphate concentrations (4 and 5) produced a clear and significant increase in intensity in this absorption band. Lower concentrations produced no changes (copper sulphate concentration 1 and 2), or low and not significant modifications (copper sulphate concentration 3). This effect is clearly related to the copper sulphate interaction with the pigment species (anthocyanins and/or co-pigments), because the red absorption tail characteristic of the pure salt was kept and did not overlap with the grape pigment absorption band. Generally, pigment-metal complexes are not considered to play significant roles in wine colour (Boulton, 2001), but these preliminary results open the way for new research. In this experiment, the copper concentrations necessary to obtain a significant effect were too high compared to the physiological and legal limits; however, it is important to consider that many other elements could produce such kinds of complexes (Takeda, 1977). Moreover, compartmentalisation in the berry skins could favour these interactions, thus providing higher concentrations limited to the cell vacuoles.

Also, if malvidin is not able to complex metals directly (Boulton, 2001; Dixon et al., 2005), these results suggest that less concentrated anthocyanins could play an important role in metal interactions to produce colour variations. Sangiovese and Cabernet Sauvignon were selected due to their typical anthocyanin profiles. Considering only 3-Oglucoside pigments, malvidin derivatives are expected to represent about $60 \%$ of the total anthocyanins in Cabernet Sauvignon (Mattivi, 2006) and 40\% in Sangiovese (Rustioni et al., 2013b). However, also considering peonidin-3-Oglucoside as non-ortho-diphenol, the pigments not able to produce metal complexes increase to about $67 \%$ in Cabernet Sauvignon (Mattivi, 2006) and 57\% in Sangiovese (Rustioni et al., 2013b). The initial anthocyanin content in the extracts was different, as demonstrated by the absorption intensity without copper sulphate addition (Fig. 2c and 2d) and, in both the cultivars, the higher copper sulphate addition produced around five times more of the initial absorption intensity at $525 \mathrm{~nm}$ (Table 1). Considering the similar effect in the two cultivars, and the huge colour increase, I suggest that complex solutions such as grape extracts could favour metal interactions involving different molecular species, includ-
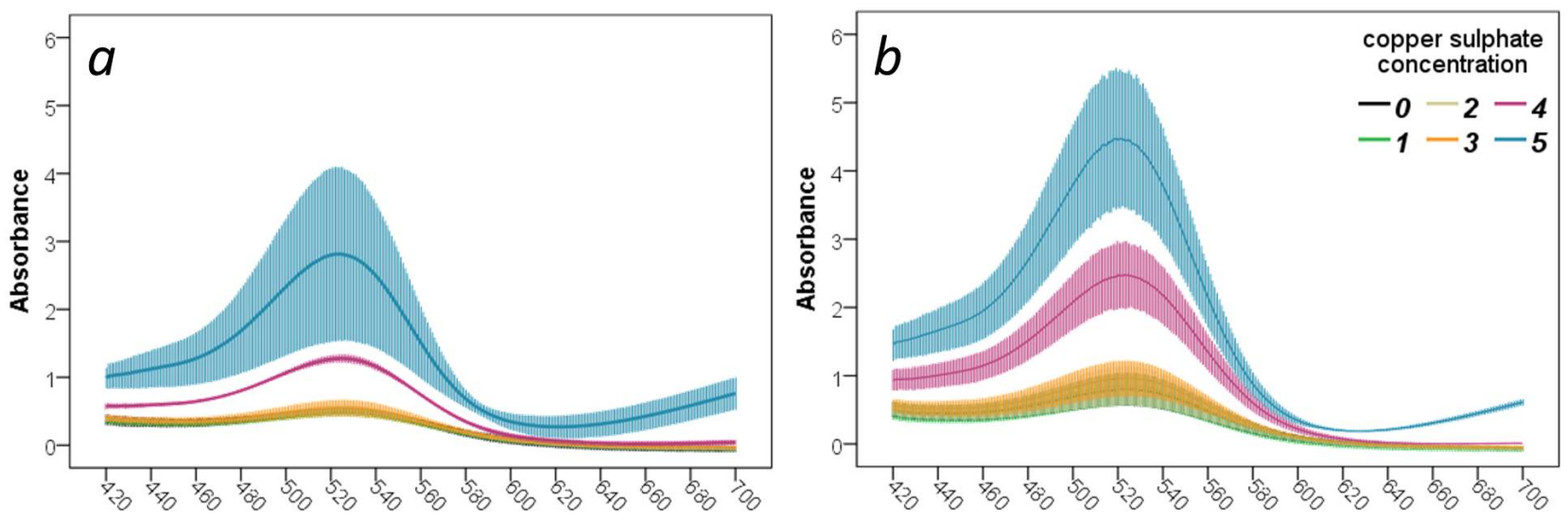

wavelength
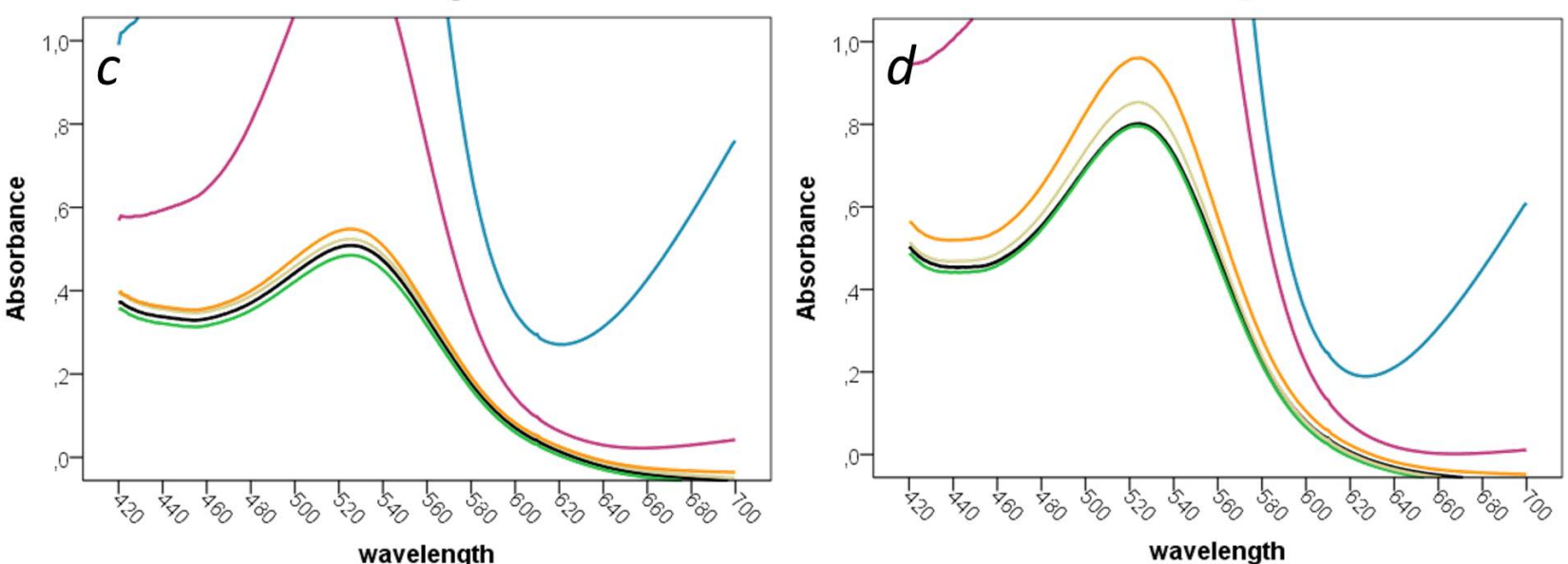

FIGURE 2

The effect of copper sulphate, at different concentrations (0 to 5), on grape extract absorption spectra. Results of Sangiovese grapes at a 95\% confidence interval (bars) (a) and details of low salt additions (c). Effect on Cabernet Sauvignon grapes at a $95 \%$ confidence interval (bars) (b) and details of low salt additions (d). 
TABLE 1

Increase in absorption intensity ratio at $525 \mathrm{~nm}$ between copper sulphate concentration 5 and 0 . Averages are not significantly different.

\begin{tabular}{lcccr}
\hline & Average & Minimum & Maximum & Standard deviation. \\
\hline Cabernet Sauvignon & 5.52 & 4.68 & 6.43 & 0.88 \\
Sangiovese & 5.57 & 5.38 & 5.76 & 0.19 \\
\hline
\end{tabular}

ing non-ortho-diphenol anthocyanins and co-pigments. To clarify this hypothesis, further experiments should be done using pure compounds. Moreover, improved knowledge at the molecular level of grape anthocyanin colours (Rustioni et al., 2014b) and interactions (Di Meo et al., 2012) could support these studies through a theoretical chemistry approach.

\section{CONCLUSIONS}

Metal-anthocyanin complexes in grapes and wines have never been considered because of the prevalence of nonortho-dihydroxylated pigments in Vitis vinifera. However, the results from this study encourage possible considerations concerning some kind of coloured interactions between grape-wine anthocyanins and metals. Further experiments should be done to clarify this effect, also considering the influence of other elements of agronomic relevance (e.g., Fe, $\mathrm{Ca}, \mathrm{Mn}, \mathrm{Zn}$ and $\mathrm{Mg}$ ).

\section{LITERATURE CITED}

Boulton, R., 2001. The copigmentation of anthocyanins and its role in the color of red wine: A critical review. Am. J. Enol. Vitic. 52(2), 67-87.

Dagostin, S., Schärer, H.J., Pertot, I. \& Tamm, L., 2011. Are there alternatives to copper for controlling grapevine downy mildew in organic viticulture? Crop Prot. 30, 776-788.

Di Meo, F., Sancho Garcia, J.C., Dangles, O. \& Trouillas P., 2012. Highlights on anthocyanin pigmentation and copigmentation: A matter of flavonoid $\pi$-stacking complexation to be described by DFT-D. J. Chem. Theory Comput. 8, 2034-2043.

Dixon, R.A., Xie, D. \& Sharma, S.B., 2005. Tansley review. Proanthocyanidins - A final frontier in flavonoid research? New Phytol. 165, 9-28.

Hanlin, R.L., Hrmova, M., Harbertson, J.F. \& Downey, M.O., 2010. Review: Condensed tannin and grape cell wall interactions and their impact on tannin extractability into wine. Aust. J. Grape Wine Res. 16, 173-188.

Harborne, J.B., 1958. Spectral methods of characterizing anthocyanins Biochem. J. 70, 22-28.
Kondo, T., Yoshida, K., Nakagawa, A., Kawai, T., Tamura, H. \& Goto, T. 1992. Structural basis of blue-colour development in flower petals from Commelina communis. Nature 358, 515-518.

Mattivi, F., Guzzon, R., Vrhovsek, U., Stefanini, M. \& Velasco, R., 2006. Metabolite profiling of grape: Flavonols and anthocyanins. J. Agric. Food Chem. 54, 7692-7702.

Robinson, G.M. \& Robinson, R., 1931. A survey of anthocyanins. Biochem. J. 25, 1687-1705.

Rustioni, L., Basilico, R., Fiori, S., Leoni, A., Maghradze, D. \& Failla, O., 2013a. Grape colour phenotyping: Development of a method based on the reflectance spectrum. Phytochem. Analysis 24(5), 453-459.

Rustioni, L., Bedgood, D.R. Jr., Failla, O., Prenzler, P.D. \& Robards, K., 2012. Copigmentation and anti-copigmentation in grape extracts studied by spectrophotometry and post-column-reaction HPLC. Food Chem. 132, 2194-2201

Rustioni, L., Di Meo, F., Guillaume, M., Failla, O. \& Trouillas, P., 2014b. Tuning color variation in grape anthocyanins at the molecular scale. Food Chem. 141, 4349-4357.

Rustioni, L., Fiori, S. \& Failla, O., 2014a. Evaluation of tannins interactions in grape (Vitis vinifera L.) skins. Food Chem. 159, 323-327.

Rustioni, L., Rossoni, M., Failla, O. \& Scienza, A., 2013b. Anthocyanin esterification in Sangiovese grapes. Ital. J. Food Sci. 25, 133-141.

Takeda, K., 1977. Metallo-anthocyanins. II. Further experiments of synthesizing crystalline blue metallo-anthocyanins using various kinds of bivalent metals. Proc. Japan Acad. 53. Ser. B. 7, 257-261

Takeda, K., 2006. Blue metal complex pigments involved in blue flower color. Proc. Jpn. Acad. Ser. B 82, 142-154.

Takeda, K. \& Hayashi, K., 1977. Metallo-anthocyanins. I. Reconstruction of commelinin from its components, awobanin, flavocommelin and magnesium. Proc. Japan Acad. 53. Ser. B. 1, 1-5.

Vasconcelos, M.T., Azenha, M. \& De Freitas, V., 1999. Role of polyphenols in copper complexation in red wines. J. Agric. Food Chem. 47, 2791-2796. 